hep-ph/9909518

\title{
Neutrino masses and mixing angles from leptoquark interactions
}

\author{
Uma Mahanta \\ Mehta Research Institute \\ Chhatnag Road, Jhusi \\ Allahabad-211019, India
}

\begin{abstract}
In this paper we show that the mixing between leptoquarks (LQ's) from different $S U(2)_{l}$ multiplets can generate a non-trivial Majorana mass matrix for neutrinos through one loop self energy diagrams. Such mixing can arise from gauge invariant and renormalizable LQ-Higgs interaction terms after EW symmetry breaking. We use the experimental indication on neutrino oscillation to find constraints on specific combinations of LQ couplings to quark-lepton pairs and to the SM higgs boson. These constraints are compared with the ones from $\pi \rightarrow e \bar{\nu}_{e}$.
\end{abstract}

PACS: 23.40.-s, 14.60.Pq, 12.40.-j, 23.40.Bw. 


\section{Introduction}

The recent neutrino data from Superkamiokande (SK) [1] has provided strong evidence for $\nu_{\mu}$ oscillating into $\nu_{\tau}$ or some species of sterile neutrinos as an explanation of the atmospheric neutrino anomaly. The observed solar neutrino deficit [2] is also probably an indication of $\nu_{e}$ oscillating into some other species of neutrinos. Results from the laboratory experiment by the LSND collaboration [3] can also probably be considered as yet another type of neutrino oscillation. Neutrino oscillation imply a non-trivial structure of the neutrino mass matrix. The extreme smallness of neutrino masses compared to quarks and charged lepton masses naturally suggests that they are zero at the tree level but are generated through higher order loop corrections. The coupling constants involved in this radiative mass generation must be small in order to reproduce the small neutrino masses. In this paper we shall adopt this viewpoint and show how the experimentally suggested neutrino masses and mixing angles could be generated at one loop level through higgs induced mixing between different leptoquark (LQ) multiplets.

Leptoquarks (LQ's) [4] occur naturally in many extensions of the SM namely grand unified models, extended technicolor models and models of quark lepton substructure which contain quarks and leptons in the same multiplet. If LQ's couple both to quark pairs and quark-lepton pairs then they mediate too rapid proton decay. To avoid this disaster and to keep the LQ interpretation interesting for low energy phenomenology it is necessary that LQ coupings to quark pairs must vanish. In order to enable LQ's to couple to quarklepton pairs in the context of the $S U(3)_{c} \times S U(2)_{l} \times U(1)_{y}$ theory, they must be color triplets or antitriplets. Further they can form a singlet, doublet or triplet representation of $S U(2)_{l}$. Just like quarks and leptons come in different generations LQ's can also carry a generation index. In this work we shall assume that the number of LQ generations is equal to that of fermions $\left(n_{g}=3\right)$. This happens in the superstring inspired $\mathrm{E}(6)$ model and also in the $\mathrm{E}(8) \times \mathrm{E}(8) \sigma$ models. The coupling constant of LQ's to q-l pairs in general can depend on the the generation indices of the LQ, the quark and the lepton. Such 
a general flavor structure for LQ couplings and their phenomenological implications were considered considered in ref. [4]. The existing flavor changing neutral current data however strongly constrains the flavor off diagonal couplings of LQ's to q-1 pairs. Besides coupling to q-l pairs, LQ's can also couple to the SM gauge bosons and to the higgs doublet. Of particular importance for this paper are the Higgs-LQ trilinear interaction terms which lead to mixing between LQ's from different $S U(2)_{l}$ multiplets after EW symmetry breaking. We shall show that this mixing among different LQ multiplets gives rise to a non-trivial lepton number violating Majorana mass matrix for neutrinos through loop corrections.

The contents of this article are divided into the following subsections. In Sec. II we present the interaction Lagrangians of singlet, doublet and triplet LQ's with q-1 pairs and with the higgs doublet. In Sec. III we derive the neutrino mass matrix that arises from the mixing of doublet and singlet LQ's. In Sec. IV we show that the mixing between doublet and triplet LQ's can also generate a non-trivial mass matrix for neutrinos. In Sec. $\mathrm{V}$ we use the experimental indication on neutrino oscillation to derive some constraints on specific combinations of LQ couplings and compare them with the existing constraints. Finally in Sec. VI we present the main conclusions of our study.

\section{Interaction Lagrangians for Leptoquarks}

We shall assume that the light LQ's present in the low energy theory arise from an underlying high energy theory that breaks down into the SM gauge group at some high energy scale $\Lambda \gg v$. Here $\mathrm{v}$ is the scale associated with electroweak symmetry breaknig. To discuss the low energy LQ phenomenology in a model independent way we shall construct its Lagrangian based on invariance under the SM gauge group and renormalizability. To satisfy the strong constraints [4] of the helicity suppressed decay $\pi \rightarrow e \bar{\nu}_{e}$ on LQ's with non chiral couplings we shall assume that each LQ couples to quarks of a particular chirality only. Consider three scalar LQ fields D, S and T with the following $S U(3)_{c} \times S U(2)_{l} \times U(1)_{y}$

assignments: $D \sim\left(3^{*}, 2,-\frac{1}{6}\right), S \sim\left(3^{*}, 1, \frac{1}{3}\right)$ and $T \sim\left(3^{*}, 3, \frac{1}{3}\right)$. The general flavor structure of their Yukawa couplings to q-l pairs are given by 


$$
\begin{aligned}
L_{1} & =\left[\sum \lambda_{i j}^{k} \bar{l}_{i}^{c} i \tau_{2} q_{j} S_{k}+\sum \lambda_{i j}^{\prime k} \bar{d}_{R i} l_{j} D_{k}^{+}\right. \\
& \left.+\sum \lambda_{i j}^{\prime \prime k} \bar{l}_{i}^{c} \tau_{a} i \tau_{2} q_{j} T_{k}^{a}\right]+h . c . \\
& =\left[\sum \lambda_{i j}^{k}\left(\bar{\nu}_{L i}^{c} d_{L j}-\bar{e}_{L i}^{c} u_{L j}\right) S_{k}+\sum \lambda_{i j}^{\prime k} \bar{d}_{R i}\left(\nu_{L j} D_{k}^{+1}+e_{L j} D_{k}^{+2}\right)\right. \\
& +\sum \lambda_{i j}^{\prime \prime k}\left(\bar{\nu}_{L i}^{c} d_{L j}+\bar{e}_{L i}^{c} u_{L j}\right) T_{k}^{3}+\sum \lambda_{i j}^{\prime \prime k} \bar{e}_{L i}^{c} d_{L j} T_{k}^{+} \\
& \left.-\sum \lambda_{i j}^{\prime \prime k} \bar{\nu}_{L i}^{c} u_{L j} T_{k}^{-}\right]+h . c .
\end{aligned}
$$

In the above $\mathrm{i}, \mathrm{j}, \mathrm{k}$ refer to the generation index of the relevant field. Repeated indices are all summed over. q and 1 are LH quark and lepton fields. $d_{R}$ is the RH down quark field. $\psi^{c}=C \bar{\psi}^{T}$ is the charge conjugated fermion field. $D_{k}^{+1}$ is the $I_{3}=\frac{1}{2}$ component of $D_{k}^{+} \cdot T_{k}^{+}=T_{k}^{1}+i T_{k}^{2}$ and $T_{k}^{-}=T_{k}^{1}-i T_{k}^{2} \cdot T_{k}^{+}, T_{k}^{-}$and $T_{k}^{3}$ have the following weak isospin $I_{3}$, and charge $Q$ assignments: $T_{k}^{+} \sim\left(1, \frac{4}{3}\right), T_{k}^{-} \sim\left(0, \frac{1}{3}\right)$ and $T_{k}^{-} \sim\left(-1,-\frac{2}{3}\right)$. Since $T_{k}^{a}$ carries $U(1)_{y}$ charge $T_{k}^{a} \neq\left(T_{k}^{a}\right)^{+}$. The above interaction terms are written in the gauge or interaction basis for the relevant fields. From the above Lagrangian it follows that $S_{i}$, $T_{i}$ and $D_{i}$ have lepton numbers of $-1,-1$ and +1 respectively. Note that since the Yukawa couplings of $D_{i}, S_{i}$ and $T_{i}$ are chiral in nature they cannot generate any neutrino mass through radiative corrections unless we add new interactions.

Besides the Yukawa couplings of the LQ's, the low energy effective Lagrangian will also contain possible higgs-leptoquark interactions. The Higgs-LQ interaction can be expressed by the following Lagrangian

$$
\begin{aligned}
L_{2} & =\left[\sum K_{i}\left(D_{i}^{+} \phi_{c}\right) S_{i}+\sum K_{i}^{\prime}\left(\phi_{c}^{+} \tau_{a} D_{i}\right) T_{i}^{+a}+h . c .\right] \\
& -\sum \mu_{\alpha}^{2}\left(\chi_{\alpha}^{+i} \chi_{\alpha}^{i}\right)+\sum h_{\alpha}^{i}\left(\phi^{+} \phi\right)\left(\chi_{\alpha}^{+i} \chi_{\alpha}^{i}\right) \\
& =\frac{v+h}{\sqrt{2}}\left[\sum K_{i} D_{i}^{1 *} S_{i}+K_{i}^{\prime} D_{i}^{2} T_{i}^{-}+K_{i}^{\prime} D_{i}^{1} T_{i}^{3}+h . c .\right] \\
& -\sum \mu_{\alpha}^{2}\left(\chi_{\alpha}^{+i} \chi_{\alpha}^{i}\right)+\sum h_{\alpha}^{i}\left(\phi^{+} \phi\right)\left(\chi_{\alpha}^{+i} \chi_{\alpha}^{i}\right)
\end{aligned}
$$


where $\phi_{c}=i \tau_{2} \phi^{*} . \quad \chi_{\alpha}^{i}$ is a collective symbol for all the LQ fields with $\alpha=1,2,3$ referring to D, S and T respectively. If the SM Higgs doublet $\phi$ and the LQ's belong to the same multiplet of some higher symmetry group then such interaction terms can naturally arise when the higher symmetry group breaks down into $S U(3)_{c} \times S U(2)_{l} \times U(1)_{y}$ at some high energy scale $\Lambda$. After EW symmetry breaking at the Fermi scale the Lagrangian $L_{2}$ will generate mixing between different LQ multiplets. In the following we shall consider the mixing between two different LQ multiplets at a time. However our results will not be very different from the more general case where all the mixing terms are present provided there is no accidental cancellation among different contributions.

\section{Neutrino mass matrix from doublet-singlet mixing}

It follows from eqn (2) that after EWSB the doublet-singlet mixing term can be written as

$$
L_{3}=\sum K_{i}\left(\frac{v+h}{\sqrt{2}}\right)\left(D_{i}^{1 *} S_{i}+S_{i}^{*} D_{i}^{1}\right)
$$

The above Lagrangian mixes $D_{i}^{1}$ with $S_{i}$ both of which carry the same charge and color quantum numbers. It can be shown that the LQ fields under consideration in the mass eigenstate basis are given by $D_{i}^{1 \prime}=\cos \theta_{i} D_{i}^{1}+\sin \theta_{i} S_{i}$ and $S_{i}^{\prime}=-\sin \theta_{i} D_{i}^{1}+\cos \theta_{i} S_{i}$. The mixing angle $\theta_{i}$ is given by $\sin \theta_{i}=\frac{\frac{v K_{i}}{\sqrt{2} b_{i}}}{\left[1+\frac{v^{2} K_{i}^{2}}{2 b_{i}^{2}}\right]^{\frac{1}{2}}}$. Here $a_{i}=m_{D i}^{2}+m_{S i}^{2}$ and $b_{i}=m_{D i}^{2}-m_{S i}^{2}$. $m_{D i}$ and $m_{S i}$ are the shifted masses after EWSB and are given by $m_{D i}^{2}=\mu_{D i}^{2}-h_{D}^{i} \frac{v^{2}}{2}$ and $m_{S i}^{2}=\mu_{S i}^{2}-h_{S}^{i} \frac{v^{2}}{2}$. The primed fields refer to the mass eigenstate basis. Their masses are given by $m_{D i}^{\prime 2}=\frac{1}{2}\left[a_{i}+b_{i}+\frac{v^{2} K_{i}^{2}}{b_{i}}\right]$ and $m_{S i}^{\prime 2}=\frac{1}{2}\left[a_{i}+b_{i}-\frac{v^{2} K_{i}^{2}}{b_{i}}\right]$. All phenomenological implications of LQ interactions must be derived in terms of fields in the mass eigenstate basis. When the interactions of $D_{i}^{1}$ and $S_{i}$ with the d- $\nu$ pair are written in terms of mass eigenstate fields we get

$$
L_{4}=\sum \lambda_{i j}^{k} \bar{\nu}_{L i}^{c} d_{L j}\left(\sin \theta_{k} D_{k}^{\prime 1}+\cos \theta_{k} S_{k}^{\prime}\right)
$$




$$
+\lambda_{i j}^{\prime k} \bar{d}_{R i} \nu_{L j}\left(\cos \theta_{k} D_{k}^{\prime 1 *}-\sin \theta_{k} S_{k}^{\prime} *\right)
$$

Note that the mixing between $D_{i}^{1}$ and $S_{i}$ induced by the the higgs doublet introduces non-chiral couplings for $D_{i}^{\prime 1}$ and $S_{i}^{\prime}$ which enables them to generate neutrino masses through radiative corrections. It can be shown that one loop self energy diagrams involving the exchange of $D_{i}^{\prime 1}$ and $S_{i}^{\prime}$ gives rise to the following Majorana mass matrix for neutrinos

$$
M_{i k} \approx \frac{N_{c}}{32 \pi^{2}} \sum_{j, l}\left[\lambda_{i j}^{l}\left(m_{d}\right)_{j} \lambda_{j k}^{\prime l}+\lambda_{j i}^{\prime l}\left(m_{d}\right)_{j} \lambda_{k j}^{l}\right] \ln \frac{m_{D l}^{\prime 2}}{m_{S l}^{\prime 2}} \sin \theta_{l} \cos \theta_{l}
$$

In the above we have neglected the matrices $D_{L}$ and $D_{R}$ that connect the quark gauge eigenstates $d_{L}$ and $d_{R}$ to their respective mass eigenstates. We would like to note first that the individual diagrams for $D^{\prime 1}$ and $S^{\prime}$ exchange are separately log divergent. But the log divergences cancel each other in the sum yielding a finite result. Second the neutrino mass matrix is symmetric in flavor space which is a requirement of its majorana nature. Third the extension of the SM considered here does not include any right handed neutrino and therefore the see saw mechanism does not play any role in generating neutrino masses here. The smallness of neutrino masses in our case has to follow from the smallness of $\lambda, \lambda^{\prime}, K_{i}$ and the loop suppression factor of $\frac{1}{16 \pi^{2}}$.

\section{Neutrino masses from doublet triplet mixing}

The mixing between doublet and triplet LQ's can also give rise to a non-trivial Majorana mass matrix for neutrinos. The mixing arises from the following LQ-Higgs interaction term

$$
\begin{aligned}
L_{5} & =\sum K_{n}^{\prime}\left(\phi_{c}^{+} \tau_{a} D_{n}\right) T_{n}^{a+}+\text { h.c. } \\
& =\frac{v+h}{\sqrt{2}}\left[\sum K_{n}^{\prime} D_{n}^{1} T_{n}^{3+}+\sum K_{i}^{\prime} D_{i}^{2} T_{i}^{-}\right]+\text {h.c. }
\end{aligned}
$$

The above Lagrangian implies that $D_{n}^{1}$ mixes with $T_{n}^{3}$ and $D_{n}^{2}$ mixes with $T_{n}^{-}$. The general flavor structure of the Yukawa couplings of $T_{n}^{a}$ to q-1 pairs is given by 


$$
\begin{aligned}
L_{6} & =\sum \lambda_{i j}^{\prime \prime k} \bar{l}_{i}^{c} \tau_{a} i \tau_{2} q_{j} T_{n}^{a}+\text { h.c. } \\
& =\left[\sum \lambda_{i j}^{\prime \prime k}\left(\bar{\nu}_{L i}^{c} d_{L j}+\bar{e}_{L i}^{c} u_{L j}\right) T_{k}^{3}+. .\right]+\text { h.c. } \\
& =\left[\sum \lambda_{i j}^{\prime \prime k}\left(\bar{\nu}_{L i}^{c} d_{L j}+\bar{e}_{L i}^{c} u L j\right)\left(T_{k}^{\prime 3} \cos \theta_{k}^{\prime}-D_{k}^{\prime 1} \sin \theta_{k}^{\prime}\right)+. .\right]+\text { h.c. }
\end{aligned}
$$

where $\theta_{k}^{\prime}$ is the mixing angle between $T_{k}^{3}$ and $D_{k}^{1}$. Proceeding as in Sec. III it can be

shown that one loop self energy diagrams involving the exchange of $D_{k}^{\prime 1}$ and $T_{k}^{\prime 3}$ generates the following mass matrix for neutrinos

$$
M_{i k} \approx \frac{N_{c}}{32 \pi^{2}} \sum_{l, j}\left[\lambda_{i j}^{\prime \prime l}\left(m_{d}\right)_{j} \lambda_{j k}^{\prime l}+\lambda_{j i}^{\prime l}\left(m_{d}\right)_{j} \lambda_{k j}^{\prime \prime l}\right] \ln \frac{m_{T l}^{\prime 2}}{m_{D l}^{\prime 2}} \sin \theta_{l}^{\prime} \cos \theta_{l}^{\prime}
$$

\section{Implications of neutrino oscillation data on LQ couplings}

The experimental data indicating neutrino oscillation can be used to find constraints on specific combinations of LQ couplings. To be specific let us consider the doublet-singlet mixing case. In general the neutrino mass matrix $M_{i k}$ is not symmetric. Since the neutrino mass matrix given by eqn (5) is symmetric it can be diagonalized by a $3 \times 3$ orthogonal matrix $U$. The matrix $U$ can be completely specified in terms of three angles $\theta_{12}, \theta_{23}$ and $\theta_{13}$. It has been shown [5] that the recent SK data, the CHOOZ data [6] and the solar neutrino data can be accomodated in a three neutrino oscillation model at 99\% C.L. for $\sin \theta_{12}=.63, \sin \theta_{23}=.71, \sin \theta_{13}=.45, \delta m_{32}^{2}=m_{3}^{2}-m_{2}^{2}=8 \times 10^{-4} e v^{2}$ and $\delta m_{21}^{2}=m_{2}^{2}-$ $m_{1}^{2}=1 \times 10^{-4} e v^{2}$. The neutrino oscillation data determines only the mixing angles and the mass squared differences leaving the overall scale for neutrino masses undetermined. If we take $m_{1}=.01 \mathrm{ev}$, the elements of the neutrino mass matrix in the flavor basis will be given by $M_{11}=.015 \mathrm{ev}, M_{12}=M_{21}=.007 \mathrm{ev}, M_{13}=M_{31}=.004 \mathrm{ev}, M_{22}=.020 \mathrm{ev}$, $M_{23}=M_{32}=.008 \mathrm{ev}$ and $M_{33}=.021 \mathrm{ev}$. We would like to emphasize that the value of $m_{1}$ cannot be chosen arbitrarily. It must satisfy the constraint from neutrinoless double beta decay which provides a bound on $M_{11}$. The present experimental upper bound on the 
effective Majorana neutrino mass in the flavor basis is given by $M_{11}<0.2-0.4 \mathrm{eV}$ at $90 \%$ CL [7]. The range of the upper bound is mainly due to the uncertainty in the theoretical calculation of the nuclear matrix elements. Besides the resulting values of $m_{2}$ and $m_{3}$ must satisfy the bounds $m_{2}<.17 \mathrm{Mev}$ and $m_{3}<18 \mathrm{Mev}$ [8]. Using the expression of $M_{i k}$ derived earlier we can now find constraints on particular combinations of LQ couplings. Since the values of $M_{i k}$ for different i,k do not differ much among them we shall consider the constraints that arise from only one of them namely $M_{11}$. We shall assume that the value of $\ln \frac{m_{D l}^{\prime 2}}{m_{S l}^{\prime 2}}$ do not change appreciably with generation. For $m_{D}^{\prime}=300 \mathrm{Gev}$ and $m_{S}^{\prime}=200 \mathrm{Gev}$ we get $\sum\left(\lambda_{1 j}^{l}\right)^{2} m_{d j} \sin \theta_{l} \cos \theta_{l} \approx .74 \times 10^{-9}$ Gev. Further if we assume that only one product coupling is non-zero at a time we get $\left(\lambda_{11}^{l}\right)^{2} \sin \theta_{l} \cos \theta_{l} \approx .74 \times 10^{-7},\left(\lambda_{12}^{l}\right)^{2} \sin \theta_{l} \cos \theta_{l} \approx$ $5.36 \times 10^{-9}$ and $\left(\lambda_{13}^{l}\right)^{2} \sin \theta_{l} \cos \theta_{l} \approx 1.48 \times 10^{-10}$ indicating a flavor dependent heirarchy in LQ couplings. If we set $M_{11}=.4 \mathrm{eV}$ which is the present upper bound from neutrinoless double beta decay we get instead $\left(\lambda_{11}^{1}\right)^{2} \sin \theta_{l} \cos \theta_{l} \approx 1.97 \times 10^{-6}$. For a LQ mass of 200 Gev, HERA and Tevatron will soon be able to probe flavor diagonal LQ coulpings for first generation down to .1 . If we set $\lambda_{11}^{1} \approx .1$ we get $K_{1} \approx 2.85 \mathrm{Mev}$ which is close to the light quark masses. It is interesting to compare the constraints on LQ couplings derived from neutrino oscillation data with those derived from other low energy experiments e.g. $\pi \rightarrow e \bar{\nu}_{e}$. The mixing between different multiplets of LQ's introduces non-chiral couplings for LQ's. Such non-chiral LQ couplings can give rise to helicity unsuppressed contributions to $\pi \rightarrow \bar{\nu}_{e}$ [8]. For the exchange of $\mathrm{S}$ we have $g_{R}=\lambda_{11}^{1} \sin \theta_{1}$ and $g_{L}=\lambda_{11}^{1} \cos \theta_{1}$. From ref[4] we then get $g_{l} g_{R}=\left(\lambda_{11}^{\prime 1}\right)^{2} \sin \theta_{1} \cos \theta_{1}<\frac{m_{S}^{2}}{(100 \text { Tev })^{2}} \approx 4 \times 10^{-6}$. Actually the contributions due to both $D_{i}^{1}$ and $S_{i}$ exchange must be taken into account which raises the bound to $8 \times 10^{-6}$. This bound is nearly two orders of magnitude greater than the value provided by the neutrino oscillation data for $M_{11}=.015 \mathrm{eV}$. However it is close to the value provided by the neutrino oscillation data for $M_{11} \sim 0.4 \mathrm{eV}$. The next generation of neutrinoless double beta decay is expected to be sensitive to values of $M_{11}$ in the range $10^{-2}-10^{-1} \mathrm{eV}$. It will enable us to decide whether the neutrino data gives more stringent 
bounds on LQ couplings than the pion decay rate.

Coming next to other bounds on flavor off-diagonal LQ couplings, we note that flavor changing radiative deacys like $\mu \rightarrow e \gamma$ require non-chiral couplings for LQ's and hence mixing between different multiplets of LQ's. To be specific consider the higgs induced mixing between $D_{i}^{1}$ and $S_{i}$. But this mixing does not contribute to $\mu \rightarrow e \gamma$ since $D_{i}^{1}$ does not couple to $e_{i}-q$ pair. Similarly the mixing between $D_{i}^{1}$ and $T_{i}^{3}$ or $D_{i}^{2}$ and $T_{i}^{-}$does not contribute to radiative muon deacy because $D_{i}^{1}$ and $T_{i}^{-}$does not couple to charged leptonquark pair. The decay $\mu \rightarrow e \gamma$ therefore does not impose any bound on the parameters of our model. We would also like to note that the LQ masses chosen by us are consistent with the latest bounds from HERA and Tevatron [9]. The HERA bounds depend on the value of $g_{l q}$. For $g_{l q}=e$ the HERA bounds are $237 \mathrm{Gev}$ for first generation and $73 \mathrm{Gev}$ for second generation. The Tevatron bounds are most stringent for first generation $(>225$ Gev) and become progressively weaker for second (>131 Gev) and third generation (> 95 Gev). The Tevatron and HERA bounds however depend on several assumptions the most crucial of which is the branching ratio of the LQ into e-q or $\nu$-q pair. The bounds get significantly relaxed if the branching ratios are lower than those usually assumed. Such reduction in branching ratios can take place in models where there is an appreciable mixing between LQ's from different $S U(2)_{l}$ multiplets.

\section{Conclusion}

In conclusion in this paper we have shown that the interaction between LQ's and the the SM higgs doublet induces mixing between LQ's from different $S U(2)_{l}$ multiplets after EW symmetry breaking. This mixing introduces small non-chiral couplings for LQ's in the mass eigenstate basis and generates a non-trivial Majorana mass matrix for neutrinos through one loop radiative correction. We have determined the neutrino mass matrix in terms of LQ couplings and masses for the singlet-doublet mixing and the doublet-triplet mixing. Using the recent combined experimental data indicating neutrino oscillation data we have derived constraints on specific combinations of LQ couplings to q-1 pairs and to 
the higgs boson. Such constraints are comparable with those derived from $\pi \rightarrow e \bar{\nu}_{e}$ if the value of $M_{11}$ is close to the present upper bound provided by the neutrinoless double beta decay.

\section{References}

1. Y. Fukuda et al., Phys. Rev. Lett. 81, 1562 (1998); hep-ex/9805006, hep-ex/9805021 and hep-ex/9807003.

2. B. T. Cleveland et al., Nucl. Phys. B (Proc. Suppl.) 38, 47 (1995); Kamiokande Collaboration, Y. Fukuda et al., Phys. Rev. Lett. 77, 1683 (1996); GALLEX Collaboration, W. Hampel et al., Phys. Lett. B 388, 384 (1996); SAGE Collaboration, J. N. Abduarashitov et al., Phys. Rev. Lett. 77, 4708, (1996); J. N. Bahcall, P. I. Kratev and A. Yu. Smirnov, hep-ph/9807216.

3. LSND Collaboration, C. Athanassopoulos et al., Phys. Rev. Lett. 75, 2560 (1995); $77,3082(1996)$.

4. W. Buchmuller, Acta. Phys. Austriaca, Suppl 27, 517 (1985); W. Buchmuller and D. Wyler, Phys. Lett. B, 177, 377 (1986); W. Buchmuller, R. Ruckl and D. Wyler Phys. Lett. B 191, 442 (1987).

5. G. L. Fogli, E. Lisi, A. Marrone and G. Sciascia, Phys. Rev. D 59, 033001 (1999).

6. CHOOZ Collaboration, M. Apolonio et al., Phys. Lett. B 420, 397 (1998).

7. L. Baudis et al., Phys. Rev. Lett. 83, 41 (1999), hep-ex-9902014.

8. M. Hirsch et al., Phys. Lett. B 378, 17 (1996); Phys. Rev. D 54, 4207 (1996).

9. Review of Particle Properties, Euro. Phys. Jour. C 3, 1 (1998). 